\title{
AN EXISTENCE THEOREM FOR OPTIMAL CONTROL WITH NONSTANDARD COST FUNCTIONALS
}

\section{R.N. MUKHERJEE and SHIV PRASAD}

\author{
Mathematics Section \\ School of Applied Sciences \\ Institute of Technology \\ Banaras Hindu University \\ Varanasi-221 005, INDIA \\ (Received September 21, 1983)
}

ABSTRACT. An existence theorem for optimal control is obtained for a general nonstandard cost functional of fractional type in this work. As an application of our result we can derive an existence theorem for optimal control given by M. B. Subrahamanyam for a cost functional, which is a ratio of two given integral cost functionals.

KEY WORDS AND PHRASES. Nonstandard cost functional, optimal control. 1980 AMS SUBJECT CLASSIFICATION CODE. 49B27, 65K10.

1. INTRODUCTION.

Consider the n-dimensional system

$$
x=A(t) x+B(t) u, x\left(t_{0}\right)=0
$$

for $t \in\left[t_{0}, t_{1}\right], t_{1}<\infty$; where $A(t)$ and $B(t)$ are matrices of size $n \times n$ and $n \times r$ respectively. We lay some further restrictions on $x(t)$ and $u(t) 1$ ater. We minimize a functional of the following type

$$
F(x, u)=\frac{F_{1}(u)}{\left[F_{2}(x)\right]^{\alpha}}
$$

where $\alpha>0$ and $\mathrm{u}$ is a measurable control. We make the following assumptions:

(a) $\mathrm{A}(\mathrm{t})$ and $\mathrm{B}(\mathrm{t})$ are continuous $\mathrm{n} \times \mathrm{n}$ and $\mathrm{n} \times \mathrm{r}$ matrix functions respectively.

(b) (i) $F_{1}($.$) is convex in u$ and $F_{1}, F_{2}$ both are continuous and

(ii) $\left|F_{1}(u)\right|<\infty$ for all admissable $u$.

(c) $\mathrm{F}_{1}(\mathrm{u}) \geqslant \mathrm{a}\|\mathrm{u}\|_{\mathrm{p}}, \mathrm{a}>0, \mathrm{p}>1$ and $\mathrm{F}_{2}(\mathrm{x}) \geqslant 0$ along any $\mathrm{x}(\mathrm{t})$ which is response to some admissable $u(t)$.

(d) For each $\mathrm{k}<\infty$ such that if $\|\mathrm{u}\|_{\mathrm{p}} \leqslant \kappa$, then

$$
\left|F_{2}(x)\right|<\infty
$$

for any admissable $u$ whose trajectory obeys any constraints imposed.

(e) There exists $k>0$ such that for every $c \geqslant 0$, 


$$
\begin{aligned}
& F_{1}(c u)=c^{k} F_{1}(u) \\
& F_{2}(c x)=c^{k / \alpha} F_{2}(x)
\end{aligned}
$$

By (1.3), this assumption implies that for every $c>0, F(c x, c u)=F(x, u)$.

(g) There exists an admissable control, the trajectory of which satisfies the imposed constraints and is such that

$$
\mathrm{F}_{2}(\mathrm{x})>0 \text {. }
$$

(h) If $\left\{u^{i}\right\}$ is a sequence of functions in $L_{p}\left[t_{0}, t_{l}\right]$ going weakly to $u_{0}$ in $L_{p}\left[t_{0}, t_{1}\right]$ then

$$
F_{1}\left(u^{o}\right) \leqslant \lim _{i \rightarrow \infty} \text { inf } F_{1}\left(u^{i}\right)
$$

We call a constraint regular if the following two conditions hold:

(1) $(x, u)$ satisfies the constraint $\Rightarrow(c x, c u)$ satisfies the constraint for every $c>0$.

(2) Let $\left(x^{1}, u^{1}\right),\left(x^{2}, u^{2}\right), \ldots$ be admissable pairs such that $u^{i} \rightarrow u^{0}$ weakly in $L_{p}\left(t_{0}, t_{l}\right)$. Suppose $\left(x^{n}, u^{n}\right)$ satisfies the constraint for each $n \geqslant 1$. Then $\left(x^{o}, u^{o}\right)$ obeys the constraint.

REMARK. Observe that in the proof of theorem 1.1 below it has been shown that $u^{0}$ is necessarily admissable.

First of all we prove the following lemma which is the modificaiton of Proposition 1.1 of [1] in the general setting.

LEMMA 1.1 .

Consider all pairs that obey (1.1) and the constraints. Assume that all the constraints are regular, and let

$$
\lambda=\inf _{(x, u)} F(x, u)=\inf _{(x, u)\left[F_{2}(x)\right]^{\alpha}}^{F_{1}(u)}
$$

( $\lambda$ is well defined by assumption (b) part (ii) and (g)). Also let

$$
\text { inf } F_{l}(u)=J \text { subject to }\left[F_{1}(x)\right]^{\alpha}=M>0 \text {. }
$$

Then $\lambda=\mathrm{J} / \mathrm{M}$.

PROOF. One can easily see that $J / M \geqslant \lambda$. To prove the reverse inequality, let u be such that $\mathrm{F}(\tilde{\mathrm{X}}, \tilde{\mathrm{u}}) \leqslant \lambda+\epsilon$ for some $\epsilon>0$. Let $\left[\mathrm{F}_{2}(\tilde{\mathrm{x}})\right]^{\alpha}=\tilde{\mathrm{M}} \quad(<\infty$ by assumption (b) part (ii), (c) and (d)) and $\mu=(M / \tilde{M})^{1 / k}$. Then $(\mu \tilde{x}, \mu \tilde{u})$ obeys all the constraints by regularity of the constraints and by assumption $(e),\left[F_{2}(\mu \tilde{x})\right]^{\alpha}=M$ and $\mathrm{F}(\mu \tilde{\mathrm{x}}, \mu \tilde{\mathrm{u}}) \leqslant \lambda+\epsilon$. By $(1.8), \mathrm{J} / \mathrm{M} \leqslant \lambda+\epsilon$, since $\epsilon$ is arbitrary, the conclusion of the lemma follows.

THEOREM 1.1.

Consider the system (1.1) and (1.2) along with assumptions (a) to (h). Also assume that the constraints on $x$ and $u$ are regular. Then there exists a control among all admissable controls that minimizes (1.2).

PROOF. By lemma 1.1 it suffices to exhibit a minimizing control over all admissable controls for which $\left[F_{2}(x)\right]^{\alpha}=M>0$ and trajectories of which satisfy (1.1) and all 
the constraints. Let $J=\inf _{u} F_{1}(u)$ subject to $\left[F_{2}(x)\right]^{\alpha}=M$. Choose $\left\{\left(x^{i}, u^{i}\right)\right\}$ such that $1 i: \eta F_{1}\left(u^{i}\right)=J$ with $\left[F_{2}\left(x^{i}\right)\right]^{\alpha}=M$ for each i. By assumption (c) $u^{i}$ $i \rightarrow \infty$

form a bounded sequence in $L_{p}\left(t_{0}, t_{1}\right)$ and hence a subsequence, still denoted by $\left\{u^{i}\right\}$, converges weakly to some $u^{0}$ in $L_{p}\left(t_{o}, t_{1}\right)$. Let $x^{o}$ be the response of $(1.1)$ to $u^{0}$. By assumption (a) and by the convergence, $x^{i}(t) \rightarrow x^{0}(t)$ for all $t \in\left[t_{0}, t_{1}\right.$ ) (see ref. [a]). By regularity of constraints, $x^{o}(t)$ obeys all the constraints. Assumption (b) implies $F_{2}\left(x^{i}\right) \rightarrow F_{1}\left(x^{0}\right)$, as $i \rightarrow \infty$. (Since $x^{i} \rightarrow x^{0}$ in $L_{p}\left(t_{0}, t_{1}\right)$ ). Since

$$
\begin{gathered}
\|\mathrm{u}\|_{\mathrm{p}} \leqslant \mathrm{K} \text { for some } \mathrm{K}<\infty \text {, By assumption (d), } \\
{\left[\mathrm{F}_{2}\left(\mathrm{x}^{\mathrm{o}}\right)\right]^{\alpha}=\lim _{\mathrm{i} \rightarrow \infty}\left[\mathrm{F}_{2}\left(\mathrm{x}^{\mathrm{i}}\right)\right]^{\alpha}=\mathrm{M} .}
\end{gathered}
$$

By assumption (h)

$$
F_{1}\left(u^{o}\right) \leqslant \lim _{i \rightarrow \infty} \inf F_{l}\left(u^{i}\right)=J
$$

From which it follows that $u_{0}$ is the minimizing control.

APPLICATION. If we specialize the functional $F(x, u)$ as given by $F(x, u)=$

$$
\frac{f_{0}^{t_{1}} \phi_{1}(x(t), t) d t}{\left[f_{0}^{t_{1}} \phi_{2}(u(t), t) d t\right]^{\alpha}}
$$

Where $\phi_{1}$ and $\phi_{2}$ satisfy the condition as given in ([1], theorem 1.1$)$ we get the existence theorem of [1] as a particular case of our thoerem.

DISCUSSIONS. The general method, what we have discussed, can be extended to cover other variants of the functional given by Subrahmanyam [1]. For example, we can consider functional of the form

$$
F(x, u)=\frac{t_{0}^{t} \phi_{1}(x(t), t) d t}{\left[f_{0}^{t} \Psi_{1}(u(t), t)\right]^{\alpha_{1}} \ldots\left[f_{0}^{t} \Psi_{n}(u(t), t) d t\right]^{\alpha} n}
$$

where $\phi_{1},\left\{\Psi_{i}\right\}_{i=1}^{n}$ all have to satisfy certain restrictions analogous to the restrictions given as in $[1]$.

\section{REFERENCES}

1. SUBRAHMANYAM, M.B. On Applications of Contro1 Theory to Integral Inequalities II, Siam J. Contro1 and Optimization, 19 (1981), 479-489.

2. LEE, E.B. and MARKUS, L. Foundations of Optimal Control Theory, John Wiley, New York, 1967.

3. SUBRAHMANYAM, M.B. Necessary Conditions for Minimum in Problems with Nonstandard Cost Functionals, J. Math. Ana1. App1. 60 (1977), 601-616.

4. SUBRAHMANYAM, M.B. On Applications of Control Theory to Integral in Equalities, J. Math. Ana1. App1. 77 (1980), 47-59. 


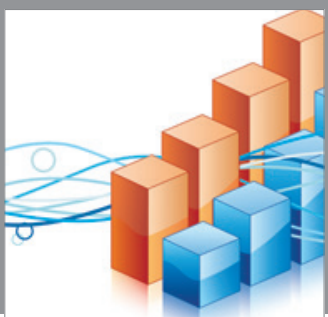

Advances in

Operations Research

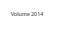

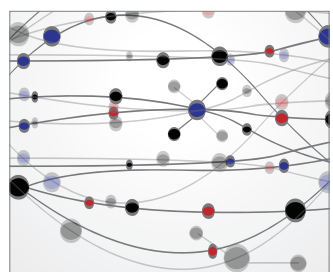

\section{The Scientific} World Journal
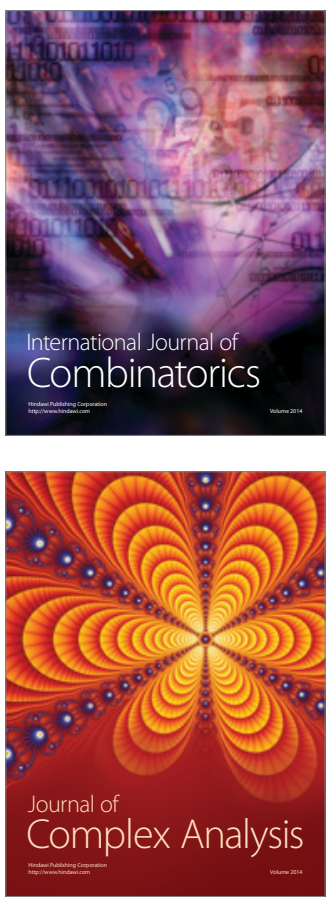

International Journal of

Mathematics and

Mathematical

Sciences
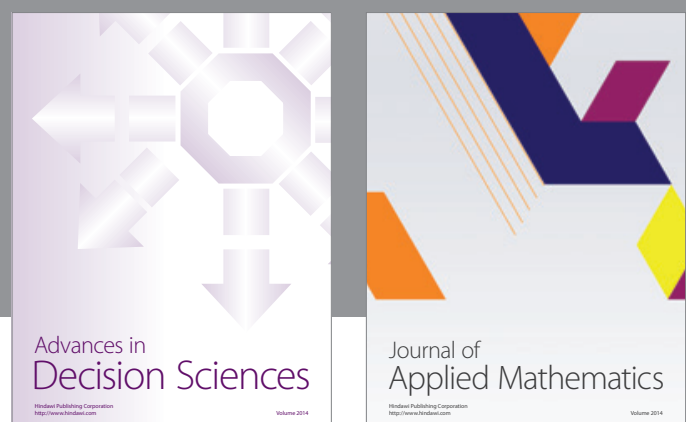

Journal of

Applied Mathematics
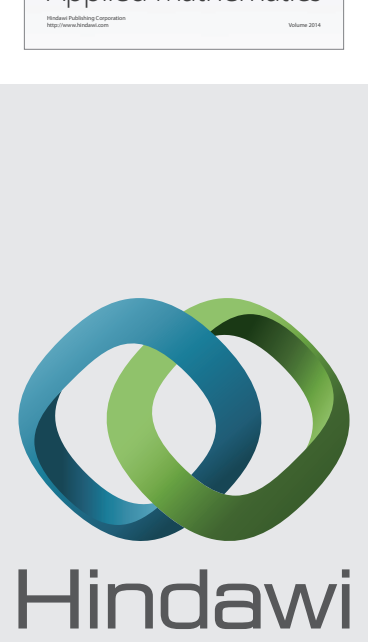

Submit your manuscripts at http://www.hindawi.com
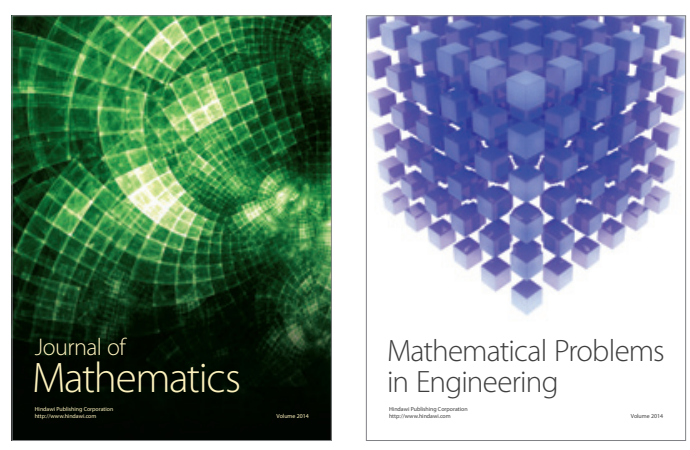

Mathematical Problems in Engineering
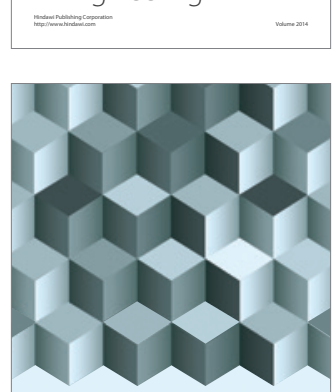

Journal of

Function Spaces
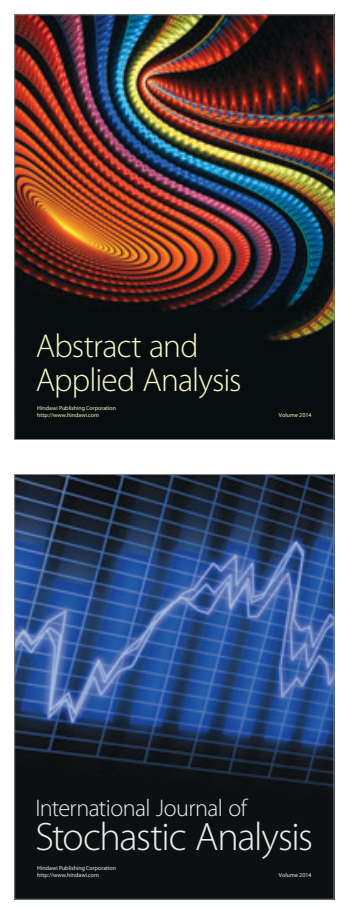

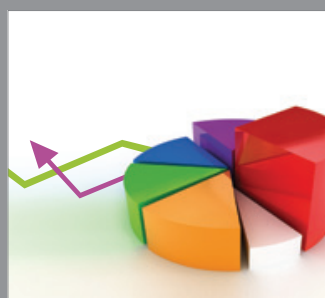

ournal of

Probability and Statistics

Promensencen
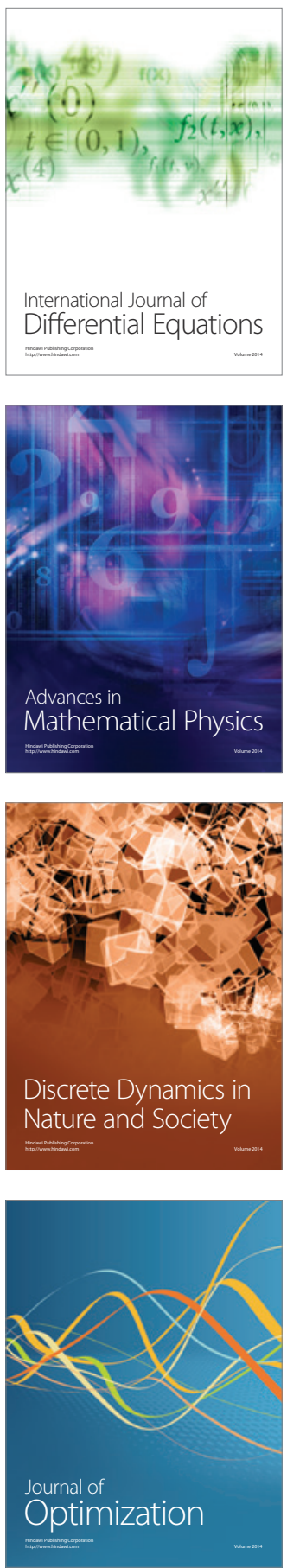